\title{
(Re)significando os projetos cuidativos da Enfermagem à luz das necessidades em saúde da população
}

\author{
Re(thinking) Nursing carative projects through the light of population health needs
}

(Re)significando proyectos cuidativos de Enfermería a la luz de las necesidades em salud de la población

\section{Maria Amélia de Campos Oliveira'}

'Universidade de São Paulo, Escola de Enfermagem, Departamento de Enfermagem em Saúde Coletiva. São Paulo-SP, Brasil.

Submissão: 14-08-2012 Aprovação: 14-08-2012

\section{RESUMO}

O conceito de necessidades é central no trabalho de Enfermagem. A Teoria das Necessidades Humanas Básicas, formulada por Wanda Horta, influenciou várias gerações de enfermeiras brasileiras e, possivelmente, ainda é a mais presente no ensino e na prática de Enfermagem no Brasil. Entretanto, há outras concepções de necessidades que podem iluminar o trabalho em saúde que, em geral, está organizado para responder a essas necessidades por meio da oferta de serviços padronizados, verticais e prescritivos. Ressignificar o cuidado em saúde e, em especial, os projetos cuidativos da Enfermagem à luz das necessidades em saúde da população requer adotar uma concepção de saúde e doença capaz de interligar aspectos individuais e coletivos.

Descritores: Enfermagem; Necessidades em Saúde.

\section{ABSTRACT}

The concept of needs is central to the work of Nursing. The Basic Human Needs Theory, formulated by Wanda Horta, influenced several generations of Brazilian nurses and possibly still is the most widespread in education and nursing practice in Brazil. However, there are other conceptions of needs that can illuminate health work that is, in general, organized to meet health needs through standardized, vertical and prescriptive service offerings. Reframing health care, specially nursing carative projects, demands to adopt a concept of health and disease capable of linking individual and collective aspects.

Key words: Nursing; Health Needs.

\section{RESUMEN}

El concepto de necesidades es fundamental para el trabajo de Enfermería. La Teoría de las Necesidades Humanas Básicas, formulada por Wanda Horta, ha influenciado a varias generaciones de enfermeras brasileñas y, posiblemente, es el más usado en la práctica y en la educación de enfermería en Brasil. Sin embargo, hay otras concepciones de las necesidades que pueden iluminar el trabajo en salud que, en general, se organiza para satisfacer las necesidades de salud a través de ofertas de servicios estandarizados, verticales y prescriptivos. El replanteamiento del cuidado en salud, especialmente los procesos cuidativos de Enfermería, demanda adoptar un concepto de salud y la enfermedad capaz de vincular aspectos individuales y colectivos.

Palabras clave: Enfermería, Necesidades en Salud. 


\section{INTRODUÇÃO}

Em 1994, a Estratégia Saúde da Família foi instituída como modelo de atenção à saúde pelo Ministério da Saúde com o objetivo de reorganizar a Atenção Básica no País e, principalmente, garantir os princípios do Sistema Único de Saúde - SUS ${ }^{(1)}$. Inicialmente recebeu o nome de Programa Saúde da Família, porém, em sua fase de consolidação, foi renomeada Estratégia Saúde da Família - ESF.

O contexto no qual a ESF foi proposta era (e ainda é) caracterizado por práticas assistenciais centradas na doença, em que a principal porta de entrada para os serviços de saúde eram (e ainda são) os hospitais. Os serviços da Atenção Básica eram considerados pouco qualificados e despreparados para o atendimento das necessidades de saúde das coletividades e pouca ênfase era dada às ações de prevenção de doenças e promoção da saúde(2).

A ESF surgiu da necessidade de transformar o modelo de atenção a saúde vigente, com foco nos princípios do SUS. Suas ações são consideradas como a principal forma de reorganização do modelo assistencial, sendo este o motivo pelo qual houve a troca do termo "Programa" para "Estratégia"(3).

A ESF assumiu a responsabilidade de fomentar o estabelecimento de vínculos e compromissos entre profissionais da saúde e população, a fim de reorganizar o modelo de atenção à saúde. Para tanto, foi necessário mudar a forma de atuação, a organização geral dos serviços e o objeto de atenção à saúde ${ }^{(4)}$.

Dentre as atribuições da ESF há ações relacionadas à promoção da saúde, atendimento da demanda espontânea e programada, prevenção do adoecimento, manutenção da saúde e atenção às fases específicas do ciclo vital ${ }^{(3)}$. Essas atribuições remetem à integralidade como princípio organizador das práticas de atenção à saúde, orientadas para as necessidades em saúde das famílias. Na ESF, a família é o foco da atenção e o espaço geossocial é o substrato para o trabalho em saúde ${ }^{(4)}$.

Redirecionar a atenção à saúde para a integralidade e a equidade implica reorganizar as práticas das equipes da ESF, de modo que tenham como objeto de atenção as famílias em um dado território geossocial e considerem as necessidades em saúde dessas famílias como necessidades de reprodução social.

\section{A centralidade do conceito de necessidades na prática de enfermagem}

O conceito de necessidades é central para o trabalho de Enfermagem. No Brasil, Wanda de Aguiar Horta formulou uma teoria para explicar a natureza da Enfermagem, definir seu campo de ação e a metodologia científica para embasar sua prática. Fundamentou-se na Teoria da Motivação Humana de Maslow, de quem adotou o conceito de necessidades humanas básicas.

Abraham Harold Maslow (1908-1970), psicólogo norteamericano de visão humanista, pesquisador do comportamento humano, considerava que os seres humanos têm necessidades comuns que motivam seu comportamento. Para ele, a motivação humana é uma tentativa de satisfação das necessidades e dos desejos das pessoas. Com base nesses princípios, propôs uma hierarquia de necessidades, organizando-as sob a forma de uma pirâmide, em cuja base estariam as necessidades fisiológicas, seguidas pelas de segurança, amor/relacionamento, estima e, no topo, as de realização pessoal. As necessidades superiores somente se manifestariam quando fossem atendidas as de ordem imediatamente inferiores.

Horta foi a primeira brasileira a formular uma teoria de Enfermagem, cujo conceito assim definiu:

é a ciência e a arte de assistir o ser humano no atendimento de suas necessidades básicas, de torná-lo independente dessa assistência, quando possível, pelo ensino do autocuidado; de recuperar, manter e promover a saúde, em colaboração com outros profissionais ${ }^{(5)}$.

Para Horta, "assistir em Enfermagem é fazer pelo ser humano aquilo que ele não pode fazer por si mesmo; ajudar ou auxiliar quando parcialmente impossibilitado de se autocuidar; orientar ou ensinar, supervisionar e encaminhar a outros profissionais" $^{\prime \prime 5)}$.

O conceito de necessidades utilizado por Horta influenciou gerações de enfermeiras brasileiras e, possivelmente, ainda é o mais presente no ensino e na prática de Enfermagem atualmente. Entretanto, há outras possibilidades de conceituar necessidades em saúde, como a apresentada a seguir.

\section{As necessidades na perspectiva Helleriana}

Em seus estudos, Campos e Mishima ${ }^{(6)}$ identificaram na literatura duas abordagens referentes ao conceito de necessidades em saúde: uma primeira, mais abstrata, presente nos trabalhos de Heller e Mendes-Gonçalves, e outra, concreto-operacional, presente nos textos de Stotz.

Segundo Heller ${ }^{(7)}$, as necessidades em saúde podem ser conceituadas como "desejo consciente, aspiração, intenção dirigida em todo momento para um certo objeto que motiva uma ação como tal. O objeto em questão é um produto social, independente do fardo de que se trate de mercadoria, de um modo de vida ou de outro homem"(7).

Duas categorias de necessidades são identificadas por Heller $^{(7)}$ : necessidades naturais e necessidades socialmente determinadas. A primeira refere-se às necessidades físicas, do campo biológico, que ela denomina necessidades necessárias. A segunda diz respeito às necessidades relacionadas a desejos conscientes, aspirações, intenções dirigidas a um objeto (proporcionado por uma objetivação social) que motiva uma ação, existindo uma correlação entre necessidades pessoais e sociais ${ }^{(7)}$.

As necessidades necessárias são necessidades naturais, próprias da espécie. Estão relacionadas ao instinto de autoconservação e inclui as necessidades de alimentação, proteção, abrigo, segurança e reprodução. Como afirma a autora, são naturalmente necessárias, pois, sem elas, os seres humanos não poderiam se manter como seres naturais.

Entretanto, Heller destaca que mesmo as necessidades naturais são necessidades sociais, pois, embora relativas à conservação e à reprodução da vida, condição para a existência humana, as formas para sua satisfação variam histórica e socialmente e elementos culturais, costumes e valores são 
decisivos para sua satisfação(7).

Heller estabelece uma distinção entre as necessidades naturais ou existenciais e as necessidades propriamente humanas. Para ela, as necessidades naturais são "ontologicamente primárias, desde o momento em que estão baseadas no instinto de autoconservação"(7). Compreendem a necessidade de alimentação, a necessidade sexual, de contato social e de cooperação e a necessidade de atividades. Ainda assim, não podem ser consideradas estritamente naturais, pois estão sujeitas a interpretações em um contexto social determinado.

Já as necessidades propriamente humanas não se limitam à garantia da sobrevivência. Compreendem o descanso superior ao necessário para a reprodução da força de trabalho, a atividade cultural, a reflexão, a amizade e a atividade moral.

Heller $^{(7)}$ faz referência ainda às necessidades alienadas, que designam as necessidades criadas pelo capitalismo, relacionadas à acumulação de riquezas. Esse grupo de necessidades apresenta uma característica quantitativa, relacionada à acumulação de capital, diferentemente das não alienadas, que têm um caráter qualitativo. A busca da satisfação das necessidades alienadas tende ao infinito e, portanto, dificilmente é alcançado um nível de saturação, pois só podem ser superadas e interrompidas por um processo de desenvolvimento das necessidades de caráter qualitativo.

Mendes-Gonçalves ${ }^{(8)}$ considerava que as necessidades alienadas só poderiam ser superadas por mudanças nas estruturas de poder que as geram. Aplicava, assim, o conceito de necessidades radicais, cuja satisfação requer a remoção de obstáculos sócio-históricos. São dirigidas para as transformações da estrutura das necessidades, "não são um tipo-ideal, mas um tipo empírico, idêntico à existência de seus portadores, que necessitam 'necessariamente' a remoção dos obstáculos sócio-históricos para sua satisfação"(8). Como não são determinadas social e historicamente, com base nos modos de produção, as necessidades radicais não são necessidades de reprodução social - estão relacionadas aos processos de criatividade e liberdade que nos tornam propriamente humanos.

Segundo Mendes-Gonçalves ${ }^{(8)}$, o ser humano é, a um só tempo, um ser natural e social, parte de uma totalidade social historicamente determinada. Possui carecimentos e potencialidades, passíveis de modificações e desenvolvimento. A partir do momento que, tanto os carecimentos como as potencialidades são formas de objetivação que se modificam e se desenvolvem, abandona-se qualquer ideia relacionada à fixidez "naturalista" de necessidades em saúde.

Para Mendes-Gonçalves ${ }^{(8)}$, o ser humano diferencia-se dos outros seres naturais justamente pela capacidade de satisfação de seus carecimentos a partir da utilização de seus poderes. Ou seja, quando lança mão de artifícios, como o pensamento ou a utilização de instrumentos para a satisfação de seus carecimentos, esses assumem um caráter social e histórico e não apenas de sobrevivência.

As necessidades humanas são, ao mesmo tempo, naturais e sociais, pois, para continuar a existir, o ser humano, como ser natural, deve relacionar-se com a natureza, por meio do trabalho. Por isso não é possível chamar de naturais quaisquer necessidades humanas, mesmo aquelas relacionadas à sobrevivência, pois, mesmo essas, para serem satisfeitas, exigem a aplicação de poderes que fazem do ser humano um ser sócio-histórico. Por essa razão, Heller ${ }^{(7)}$ passou a designá-las necessidades "necessárias".

Mesmo as necessidades "necessárias" não são fixas; modificam-se, diversificam-se e ampliam-se a cada vez que são satisfeitas. Necessidades são, portanto, produtos históricos, uma vez que, ao serem satisfeitas, permitem ao ser humano reproduzir-se como tal e carregam consigo características das primeiras necessidades satisfeitas.

Ricardo Bruno Mendes Gonçalves foi responsável pela transposição da perspectiva helleriana de necessidades para a saúde. Para ele, a satisfação de uma necessidade é o produto de um processo de trabalho. O trabalho resulta da aplicação da ação dos seres humanos sobre um objeto determinado, em resposta a uma necessidade que, ao ser reiterada ou ampliada, dá origem a outro processo de trabalho, caracterizando dessa forma a circularidade existente entre necessidades e processos de trabalho. Em suas próprias palavras: "a sócio-historicidade dos processos de trabalho cria os objetos para as necessidades e as novas necessidades criam os sujeitos para os novos processos de trabalho"(8).

Para $\operatorname{Stotz}^{(9)}$, as necessidades em saúde são individuais, biológicas, social e historicamente determinadas e satisfazê-las socialmente é um sinal do seu reconhecimento. Destaca que, embora as necessidades em saúde sejam sentidas individualmente, o sistema social de atenção procura considerá-las por meio de questões abstratas, baseadas em indicadores, por exemplo. Ao considerar apenas alguns indicadores, acaba por descontextualizar as pessoas dos determinantes de seu processo saúde-doença, privilegiando determinadas condições de saúde e ocultando ou reprimindo outras necessidades de saúde.

Cecílio(10), tal como Stotz ${ }^{(9)}$, considera que as necessidades em saúde só podem ser captadas no âmbito individual. Advoga que, adotar as necessidades como eixo norteador das práticas em saúde, exige a construção de novos saberes para reorganizar o processo de trabalho, gestão e planejamento em saúde, na luta pela equidade e pela integralidade. Para isso, destaca a necessidade de conceituar necessidades em saúde de forma que os trabalhadores da área possam se apropriar do conceito e implementá-lo no cotidiano do trabalho em saúde. Propõe uma taxonomia de necessidades em saúde, agrupando-as em quatro grandes grupos: boas condições de vida, acesso às tecnologias, criação de vínculos e autonomia.

O primeiro grupo, boas condições de vida, está relacionado tanto aos aspectos mais funcionalistas, enfatizados por fatores ambientais e externos (higiene e urbanização, por exemplo), quanto pelas diferentes posições ocupadas pelos indivíduos no modo de produção das sociedades, que melhor explicam o processo saúde-doença.

O segundo grupo relaciona-se ao acesso e consumo das tecnologias de saúde utilizadas para melhorar e prolongar a vida. O autor parte do conceito de tecnologias leve, leve-dura e dura, proposto por Merhy, ressaltando que não tem a pretensão de hierarquizá-las. Porém, destaca que o poder de uso de cada tecnologia deve estar relacionado às necessidades de cada pessoa. 
O terceiro grupo diz respeito à criação de vínculos entre usuários e profissionais de saúde, objetivando o desenvolvimento de uma relação de confiança. O vínculo é entendido como mais que uma formalidade, pois implica um encontro de subjetividades, uma relação de confiança contínua, pessoal e intransferível.

E, por fim, o quarto grupo, que diz respeito à necessidade que cada pessoa apresenta em relação à autonomia para andar a vida e que implica mais que informação e educação, pois exige dos sujeitos a reconstrução dos sentidos de sua vida, tendo essa ressignificação um peso efetivo em seu modo de viver, bem como na satisfação de suas necessidades de forma ampliada.

\section{A organização dos serviços de saúde em resposta às ne-} cessidades dos usuários

Campos e Bataiero $^{(11)}$ destacaram a complexidade que o tema das necessidades assume no âmbito dos serviços de saúde. Segundo os autores, as necessidades em saúde vêm sendo abordadas sob a perspectiva operacional, ou seja, aquela em que o objeto da atenção em saúde é conformado pela oferta dos serviços de saúde. Essa organização traz como consequência a associação das necessidades em saúde com o consumo dos serviços, geralmente sob a forma de consultas médicas.

As equipes da ESF possuem conhecimento impreciso sobre as necessidades em saúde das famílias sob seus cuidados e não dispõem de estratégias para identificar situações de vulnerabilidade a que tais famílias estão expostas. As ações das equipes em geral restringem-se à execução de atividades previstas nos programas verticais de saúde preconizados pelo Ministério da Saúde (MS), como, por exemplo, os de saúde da mulher, saúde da criança, grupos de hipertensos e diabéticos, dentre outros.

Tais programas, quando estruturados e executados de forma vertical, não atendem às necessidades em saúde das coletividades, pois não respondem à complexidade dos determinantes dos processos saúde-doença, modulados por condicionantes de ordem biológica, psicológica, cultural, econômica e política ${ }^{(12)}$.

O conceito de necessidade em saúde que subjaz às ações da equipe, focado em agravos, demandas por serviços especializados e protocolos do Ministério da Saúde, é fruto de uma concepção de saúde como ausência de doença, resultando em atividades utilitaristas e reducionistas dos fenômenos do processo saúde-doença.

Essa realidade traduz uma contradição entre as premissas do Sistema Único de Saúde (SUS) e as práticas dos serviços. Por um lado, é possível observar que o processo saúde-doença ainda é compreendido apenas no seu caráter biológico e que as ações de saúde são realizadas de forma padronizada, vertical e prescritiva. Por outro, existe uma política que visa redirecionar o sistema para as necessidades em saúde das coletividades e que busca superar o modelo médico hegemônico vigente nos serviços de saúde.

Serviços organizados de forma a responder às necessidades em saúde da população devem adotar uma concepção de saúde e doença capaz de interligar os aspectos individuais aos coletivos. Os trabalhadores de saúde devem levar em conta que as necessidades, uma vez satisfeitas, serão reiteradas ou ampliadas, o que exigirá a instauração de um novo processo de trabalho para continuar a atendê-las.

Pensar em necessidades em saúde como necessidades de reprodução social implica adotar, implícita ou explicitamente, a concepção de saúde e de doença que lhe é correspondente, no caso, a determinação social de processo saúde-doença, relacionada aos processos de produção e reprodução social ${ }^{(9,13)}$.

As necessidades em saúde diferenciam-se entre si a depender das diferentes formas de inserção social de indivíduos, famílias e grupos sociais. Estão relacionadas e expressam diferentes condições de vulnerabilidade e requerem projetos de intervenção distintos ${ }^{(8,14)}$. Portanto, satisfazê-las exige compreender as formas de viver de indivíduos e famílias.

\section{SÍNTESE}

As necessidades abrangem as múltiplas dimensões da vida (física, psíquica, afetiva, social, cultural e política). Quando validadas socialmente, são reconhecidas como demandas ou problemas de saúde e traduzidas por meio da linguagem pública em leis, normas, políticas, instituições e reinvindicações de grupos sociais ${ }^{(15)}$.

A qualidade de vida e saúde depende da satisfação tanto das necessidades necessárias (de sobrevivência e reprodução), quanto das propriamente humanas, de proteção, afeto, compreensão, acesso a educação e cultura, lazer, participação, criação, identidade, liberdade, relacionadas à autorrealização, baseadas na igualdade e no livre e mútuo reconhecimento da condição humana.

Já as necessidades alienadas são criadas em função do capital; referem-se, sobretudo, a aspectos materiais e ao consumo de mercadorias; reforçam a insatisfação (pela criação infinita de necessidades), expressa como carecimento (falta de algo, geralmente material); são inesgotáveis e, no capitalismo, parte integrante da subjetividade (sentidos, motivações).

O ser humano e suas necessidades definem-se a partir de sua existência concreta, pois as potencialidades humanas só se efetivam na vida, no processo produtivo, nas relações. As necessidades relativas à condição humana são indispensáveis à humanização.

As necessidades em saúde têm tanto valor de uso (intervenções) como valor de troca (produtos, mercadorias) e são manipuladas socialmente (criadas e ocultadas). Os serviços de saúde são contextos instauradores de necessidades (estabelecem demandas e consumo), pois o saber técnico e a ação profissional reproduzem e instauram necessidades em saúde. Os usuários participam desse contexto instaurador de necessidades, com suas demandas.

Necessidades não são somente carecimentos (falta de algo). Pensá-las apenas como carências nega a existência de necessidades que expressam potencialidades humanas. Mas as carências, traduzidas em demandas, buscam reconhecimento social e também contribuem para a autodeterminação humana. Portanto, necessidades e carências devem ser igualmente reconhecidas, pois ambas são legítimas. 


\section{REFERÊNCIAS}

1. Ministério da Saúde (Brasil), Secretaria de Atenção à Saúde. Departamento de Atenção Básica. Saúde da família no Brasil: uma análise de indicadores selecionados: 1998-2005/2006. Brasília: Ministério da Saúde; 2008.

2. Secretaria de Políticas de Saúde (Brasil), Departamento de Atenção Básica. Programa de Saúde da Família: informes técnicos institucionais. Rev Saúde Pública 2000;34(3):316-9.

3. Ministério da Saúde (Brasil). Portaria GM 648, 28 de março de 2006. Aprova a Política Nacional de Atenção Básica, estabelecendo a revisão de diretrizes e normas para a organização da Atenção Básica para o Programa Saúde da Família (PSF) e o Programa Agentes Comunitários de Saúde (PACS). Diário Oficial da União 29 mar 2006.

4. Ministério da Saúde (Brasil), Secretaria de Assistência à Saúde. Coordenação de Saúde da Comunidade. Saúde da Família: uma estratégia para a reorientação assistencial. Brasília: Ministério da Saúde; 1997.

5. Horta WA. Processo de Enfermagem. São Paulo: EDUSP; 1979. p. 1-99.

6. Campos CMS, Mishima SM. Necessidades de saúde pela voz da sociedade civil e do Estado . Cad Saúde Pública 2005;21(4):1260-68.

7. Heller A. Una revisión de la teoría de las necesidades. Barcelona: Ediciones Paidós; 1996.

8. Mendes-Gonçalves RB. Práticas de saúde: processos de trabalho e necessidades. São Paulo: Centro de Formação dos Trabalhadores em Saúde da Secretaria Municipal de Saúde de São Paulo. 1992. (Cadernos CEFOR, 1 - Séries Textos).
9. Stotz EN. Os desafios para o SUS e a educação popular: uma análise baseada na dialética da satisfação das necessidades de saúde. In: Rocha CMF, Santos L, Bilibio LFS, Ceccim RB, Feuerwerker HAP, organizadores. Ver-SUS Brasil: cadernos de textos. Brasília: Ministério da Saúde; 2004. p. 284-99.

10. Cecílio LCO. As necessidades de saúde como conceito estruturante na luta pela integralidade e eqüidade na atenção em saúde. In: Pinheiro R, Mattos RA, organizador. Os sentidos da integralidade na atenção e no cuidado à saúde. Rio de Janeiro: Abrasco; 2001. p. 113-25.

11. Campos CMS, Bataiero MO. Necessidades de saúde: uma análise da produção científica brasileira de 1990 a 2004. Interface Comun Saúde Educ 2007;11(23):605-18.

12. Ministério da Saúde (Brasil) Secretaria de Atenção à saúde. Departamento de regulação, Avaliação e Controle de Sistemas. Coordenação de Programação da Assistência. Diretrizes para a programação pactuada e integrada da assistência à saúde. Brasília: Ministério da Saúde; 2006.

13. Laurell AC. A saúde-doença como processo social. In: Nunes ED, organizador. Medicina social: aspectos históricos e teóricos. São Paulo: Global; 1983. p. 133-58.

14. Nakamura E, Egry EY, Campos CMS. et al. O potencial de um instrumento para o reconhecimento de vulnerabilidades sociais e necessidades de saúde: saberes e práticas em saúde coletiva. Rev Latino-am. Enferm 2009; 17(2):253-58.

15. Teixeira CF. O futuro da prevenção. Salvador: Casa da Qualidade Editora; 2001. p. 114. 